Tohoku J. exp. Med., 1981, 133, 1-19

\title{
Congenital Factor X Deficiency in Japan
}

\author{
Kazuo Mori, Hideaki Sakai, Noboru Nakano, Sozo Suzuki, \\ Koji Sugai, Shizue Hisa and Yoshio Goto
}

The Third Department of Internal Medicine, Tohoku University School of Medicine, Sendai 980

Mori, K., Sakai, H., Nakano, N., Suzuki, S., Sugai, K., Hisa, S. and Goto, Y. Congenital Factor $X$ Defficiency in Japan. Tohoku J. exp. Med., 1981,133 (1), 1-19 C Congenital factor $\mathrm{X}$ deficiency is a very rare inherited coagulation abnormality. There have been reported 43 cases of this disorder in the world and only 2 cases in Japan. Recently, we have hemostatically and immunologically examined as many as 3 cases of this rare deasese, 18-year-old male, 11-year-old male and 6-year-old female. Hemostatic examinations revealed prolonged prothrombin time, partial thromboplastin time and decreased serum thromboplastic activity in these 3 cases. Stypven-cephalin clotting time was also abnormal. Factor $\mathrm{X}$ activities of our cases were low when assayed by either tissue thromboplastin and partial thromboplastin or by Stypvencephalin mixture, which were $2.6,1.5$ and $4.5 \%$, respectively. The half lives of infused factor $\mathrm{X}$ were $24,38.6$ and $56 \mathrm{hr}$, respectiely, which are consistent with the data of other authors. Immunological assay of factor $\mathrm{X}$ (radioimmunoassay) showed $0.47 \mu \mathrm{g} / \mathrm{ml}$ in the second case and $0.15 \mu \mathrm{g} / \mathrm{ml}$ in the third case, from which our cases were considered to be classical factor $\mathrm{X}$ deficiency.

congenital factor X deficiency; Prower defect; abnormal factor $\mathrm{X}$ disorder; autosomal recessive inheritance; replacement therapy

Since the report of Telfer et al. (1956) on "a new coagulation defect" and the report of Hougie et al. (1957) on "Stuart clotting defect", 43 cases of congenital factor $\mathrm{X}$ deficiency have thus far been reported in the Western countries. In Japan, however, the first such case was reported by Yasunaga et al. (1964) and only one other case has since appeared (Shirakawa et al. 1969). We have experienced 3 cases, each of which was reported at the time (Mori et al. 1972, 1975a, 1976). Here we dissuss these cases together with a review of the world literature, including the Japanese cases, of congenital factor $\mathrm{X}$ deficiency.

\section{Case Presentation}

Case 1 (18-year-old male)

Anamnesis. No abnormalities were indicated throughout the prenatal period or early childhood, but at the age of 4 a large hematoma was formed on the face and head due to trauma. Thereafter, mild epistaxis was experienced and at 7 years of age major nasal bleeding occurred, demanding blood transfusion. At the ages of 9 and 11, swelling of the limbs, thought to be intramuscular hematoma, was experienced, but his condition

Received for publication, August 17, 1979.

This paper was presented at the 4lst Annual Meeting of the Japanese Society of Haematology, held in Tokyo on April 5-6, 1979. 
improved only with bed rest and wet packs. Following entrance into junior high school, swelling of the limbs and head occurred yearly, causing severe pain. He was hospitalized and treated with blood transfusion. Having finished junior high school, he left school but was hospitalized 2 months later due to a leg wound, followed by hemorrhage, swelling and epistaxis. He was then referred to our Department for further examination.

Family history. Both parents were healthy, without consanguinity on either side. One of the 6 siblings died 3 days after birth with unknown cause. All of the other siblings are healthy without signs of hemorrhagic tendencies (Fig. 1).

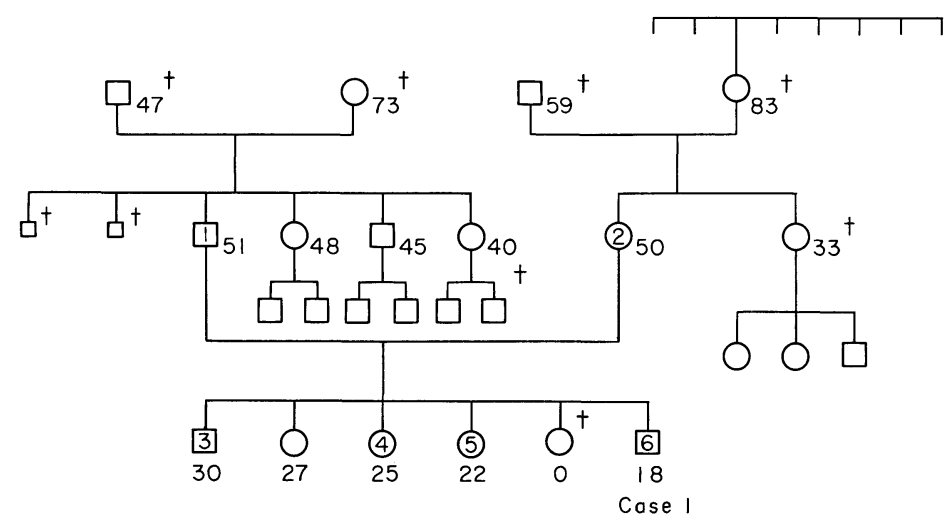

Fig. 1. Pedigree of Case 1. Figures indicate ages in years.

Physical examinations. No abnormalities. Bones and joints were also normal.

Laboratory examinations. No abnormalities (Table 1).

Hemostatic examinations. As shown in Table 6, bleeding time was normal; whole blood clotting time was slightly prolonged (15 min $30 \mathrm{sec}$ ); and thrombelastgram (TEG) showed prolongation of $r$ and $k(r=53.5 \mathrm{~mm}, k=20.5 \mathrm{~mm})$. No abnormalities of capillary resistance, clot retraction or platelet count were found, but kaolin partial thromboplastin time (K-PTT) was markedly prolonged $(89 \mathrm{sec})$. Moreover, the one stage prothrombin time (PT) and thrombotest (TT) showed marked hypoactivity. The thromboplastin generation test (TGT) indicated no abnormalities of adsorbed plasma or platelets but abnormality of serum. Determination of the activity of various clotting factors showed factors V, VIII and IX to be normal, but decreased activity of factor VII complex and of factor X. A cross-matching test with known patient's plasma (Table 2) showed that, by mixing equal volumes of patient's plasma and known hemophilia A plasma, there was marked correction of K-PTT, PT and TT. Similar results were seen on a cross-matching test with hemophilia B and Factor V deficiency plasma. Addition of hemophilia B serum to patient's plasma showed complete correction of K-PTT, partial correction of PT, but no correction of TT. It was consequently concluded that the deficient factor was other than factors V, VIII and IX. Since PTT was prolonged and PT and TT activity decreased and, moreover, the deficient factor was found in hemophilia B serum, a deficiency of factor X was indicated. Together with the determination of each of the factors separately, a diagnosis of factor $\mathrm{X}$ deficiency was made.

Effects of intravenous administration of factor $X$ concentrate. An investigation was made of the effects brought about by transfusion of a concentrate of factors II, VII, IX and X (PPSB-Nichiyaku, Nihon Pharmaceuticals). Following intravenous administration of 4 vials, changes in activity were observed. The activity of factor $\mathrm{X}$ was maintained at greater than $100 \%$ for $6 \mathrm{hr}$ immediately following administration. The activity was still at $86 \%$ after $24 \mathrm{hr}$ and $20 \%$ at $48 \mathrm{hr}$, indicating a half-life of the second phase of roughly $24 \mathrm{hr}$. PT and TT indicated activity roughly parallel to that of factor X. 
TABLE 1. Results of laboratory examinations on admission to our Department

\begin{tabular}{|c|c|c|c|c|}
\hline & & Case 1 & Case 2 & Case 3 \\
\hline $\begin{array}{l}\text { Urine and stool } \\
\text { Sedimentation rate }\end{array}$ & $\begin{array}{ll}1 \mathrm{hr} & (\mathrm{mm}) \\
2 \mathrm{hr} & (\mathrm{mm})\end{array}$ & $\begin{array}{c}\text { Negative } \\
7 \\
21\end{array}$ & $\begin{array}{c}\text { Negative } \\
60 \\
91\end{array}$ & Negative \\
\hline Liver function & $\begin{array}{l}\text { TTT } \\
\text { ZTT } \\
\text { I.I. } \\
\text { ALP } \\
\text { GOT } \\
\text { GPT }\end{array}$ & $\begin{array}{c}3.1 \\
7.9 \\
5 \\
11.6 \\
3 \\
2\end{array}$ & $\begin{array}{c}3.2 \\
11.4 \\
4 \\
15.1 \\
39 \\
10\end{array}$ & $\begin{array}{c}4.0 \\
3.6 \\
4 \\
19.2 \\
21 \\
8\end{array}$ \\
\hline Serum protein & 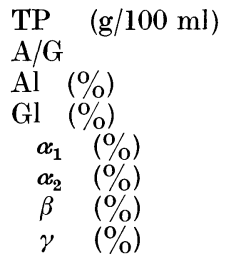 & $\begin{array}{r}7.7 \\
2.2 \\
69.3 \\
30.7 \\
3.1 \\
7.2 \\
7.1 \\
12.6\end{array}$ & $\begin{array}{r}7.7 \\
1.2 \\
54.6 \\
45.4 \\
4.4 \\
9.6 \\
10.7 \\
20.7\end{array}$ & $\begin{array}{r}7.4 \\
1.5 \\
60.5 \\
39.5 \\
5.0 \\
12.6 \\
10.1 \\
11.4\end{array}$ \\
\hline Serum electrolyte & $\begin{array}{ll}\mathrm{K} & \text { (mEq/liter) } \\
\mathrm{Ca} & \text { (mEq/liter) } \\
\mathrm{Na} & \text { (mEq/liter) } \\
\mathrm{Cl} & \text { (mEq/liter) }\end{array}$ & $\begin{array}{r}4.0 \\
5.9 \\
141 \\
101\end{array}$ & $\begin{array}{r}4.0 \\
4.5 \\
136 \\
98\end{array}$ & $\begin{array}{r}4.3 \\
4.6 \\
139 \\
105\end{array}$ \\
\hline Serum lipids & $\begin{array}{l}\mathrm{TG} \quad(\mathrm{mg} / 100 \mathrm{ml}) \\
\mathrm{PL} \quad(\mathrm{mg} / 100 \mathrm{ml}) \\
\mathrm{TC} \quad(\mathrm{mg} / 100 \mathrm{ml})\end{array}$ & $\begin{array}{r}83 \\
167 \\
160\end{array}$ & $\begin{array}{r}57 \\
143 \\
156\end{array}$ & \\
\hline Blood picture & $\begin{array}{l}\text { RBC }\left(\times 10^{4} / \mathrm{mm}^{3}\right) \\
\text { WBC }\left(\times 10^{4} / \mathrm{mm}^{3}\right) \\
\text { Hb }(\%) \\
\text { CI } \\
\text { Ret. }(\%) \\
\text { Plt.C }\left(\times 10^{4} / \mathrm{mm}^{3}\right) \\
\text { Nph. }(\%) \\
\text { Eph. }(\%) \\
\text { Bph. }(\%) \\
\text { Mon. }(\%) \\
\text { Ly. }(\%)\end{array}$ & $\begin{array}{l}567 \\
5500 \\
85 \\
0.76 \\
15 \\
24 \\
45 \\
1 \\
0 \\
3 \\
51\end{array}$ & $\begin{array}{l}440 \\
6300 \\
72 \\
0.82 \\
6 \\
30.1 \\
66 \\
0 \\
0 \\
3 \\
31\end{array}$ & $\begin{array}{c}420 \\
3400 \\
63 \\
0.71 \\
\\
22 \\
53 \\
3 \\
1 \\
4 \\
39\end{array}$ \\
\hline Congo red index & $(\%)$ & 61 & & \\
\hline
\end{tabular}

Family study. With the exception of one elder sister, abnormalities were found in all 4 other family members tested (Table 3).

Chromosome study. The chromosome number was 46 in $96.3 \%$ of the cells. With regard to karyotype, the relative length of $\mathrm{X}$ chromosomes and autosomes was slightly shorter, and the centromere index of autosomes was slightly increased.

Case 2 (11-year-old male)

Anamnesis. Immediately following the birth, umbilical hemorrhage could not be halted and it continued for 6 months. Subcutaneous hemorrhage due to injury and hematoma formation were frequently seen. Following incision of a hematoma at the age of 3 , hemostasis did not occur and he was given his first blood transfusion under the diagnosis of hemorrhagic diathesis. From that time epistaxis was frequently experienced and at the age of 5, massive blood transfusion was given due to hemoptysis and hematemesis. Upon entering primary school at the age of 6 , swelling of the right knee was seen and frequently recurred. Recovery followed blood transfusion and application of wet packs. He was 
TABLE 2. Results of crossmatching test (Kaolin PTT)

\begin{tabular}{|c|c|c|}
\hline & Case 1 & Case 2 \\
\hline Control plasma & 37.4 sec & $34.6 \mathrm{sec}$ \\
\hline Patient plasma & 89.1 & 106.5 \\
\hline Hemophilia A plasma & 92.9 & 83.4 \\
\hline Hemophilia B plasma & 117.6 & 101.1 \\
\hline Parahemophilia plasma & 249.0 & \\
\hline Patient plasma +hemophilia A plasma & 43.1 & 43.0 \\
\hline Patient plasma +hemophilia B plasma & 49.9 & 47.5 \\
\hline Patient plasma + parahemophilia plasma & 38.3 & \\
\hline Patient plasma + hemophilia B serum & 31.3 & \\
\hline Hemophilia A plasma +hemophilia B plasma & & 45.9 \\
\hline Patient plasma+normal $\mathrm{BaSO}_{4}$ plasma & & 94.4 \\
\hline Patient plasma + normal serum & & 31.0 \\
\hline Patient plasma + Coumarin plasma & & 41.6 \\
\hline Case 1 plasma+Case 2 plasma & \multicolumn{2}{|c|}{$98.5 \mathrm{sec}$} \\
\hline
\end{tabular}

TABLE 3. Data of other family members of Case 1

\begin{tabular}{|c|c|c|c|c|c|}
\hline & KPTT (sec) & PT $(\%)$ & $\mathrm{TT}(\%)$ & VII (\%) & $\mathrm{X}(\%)$ \\
\hline Father & 37.4 & 80 & 46 & 70 & 86 \\
\hline Mother & 35.2 & 71 & 43 & 62 & 16 \\
\hline Brother & 39.3 & 92 & 41 & 82 & 15 \\
\hline Sister & 37.0 & 94 & 54 & 50 & \\
\hline Sister & 35.3 & 96 & 100 & 90 & 100 \\
\hline
\end{tabular}

treated by an internist for one month for appendicitis at the age of 7 or 8 , but since it was later found that bending at the hip was difficult, it is thought that intramuscular hemorrhage of the iliopsoas muscle was the cause of the appendicitis-like pain. From 1970, swelling of the right knee subsided, but was followed by bilateral foot and left knee swelling. From October of 1971, swellings of the right hip and left knee were apparent and he complained of moderate pain.

Family studies were limited due to parental divorce, but neither hemorrhagic diathesis nor consanguinity was indicated.

Physical examinations. Intramuscular hematoma of the right hip and swelling of the left knee were seen. The patient complained of mild pain, but there were no other notable findings.

$X$-Ray findings of the bone and the joint. Pathological changes of the right knee were apparent, but they differed from those of hemophilia. Marked destruction of the chondylen itself, rather than the fossa interchondyloidea, bone atrophy of the epiphysis, and widening of the epiphyseal line due to pressure from above were apparent. Annular sclerotic changes of the metaphysis and destruction of the femuro-patellar joint surface were marked.

Laboratory examinations. Blood sedimentation rate was moderately accelerated and there was mild hypochromic anemia, but no other abnormalities were found (Table 1).

Hemostatic examinations. As shown in Table 6, bleeding time was $10 \mathrm{~min}$ and whole blood clotting time was slightly prolonged $(20 \mathrm{~min} 30 \mathrm{sec})$. TEG indicated prolonged $r$ and $k$ values. Platelet count was normal, but K-PTT, PT and TT were markedly prolonged and TGT indicated serum hypoactivity. No increase in fibrinolysis was seen or no circulating anticoagulants detected. Marked decreases in clotting factor $\mathrm{X}$ were seen and the activity of all other factors was similar to that of our first case. The patient was diagnosed as having a congenital defect in clotting factor $\mathrm{X}$. A cross-matching test with a patient with known clotting factor deficiency patients (Table 2) showed a correcton for both hemophilia A and hemophilia B plasma, but no correction with the preceding case. 
Correcting effect was not found with adsorbed normal plasma, but incomplete correction was seen with normal serum and coumarin treated patient's plasma. These results also supported the diagnosis of factor $\mathrm{X}$ deficiency.

Effects of intravenous administration of factor $X$ concentrate. As in Case 1, 2 vials of PPSB-Nichiyaku (Nihon Pharmaceuticals) were administered intravenously. TEGs at this time showed complete correction. Disappearance curve of infused factor $\mathrm{X}$ showed biphasic pattern with time, and the half-life of the second phase was $38.6 \mathrm{hr}$, i.e., 1.6 days (Fig. 2).

Chromosome study. The characteristics of chromosomes obtained from peripheral blood were as follows: $65 \%$ had 46 , and $35 \%$ had 45 chromosomes. No significant changes were seen in the nuclear shape of the nuclei containing 46 chromosomes, but in those containing 45, one of the No. 12 autosomes was missing (Fig. 3). That is, a large percent (35\%) of the nuclei showed 45 chromosomes, indicating a mosaic of 45 and 46 .

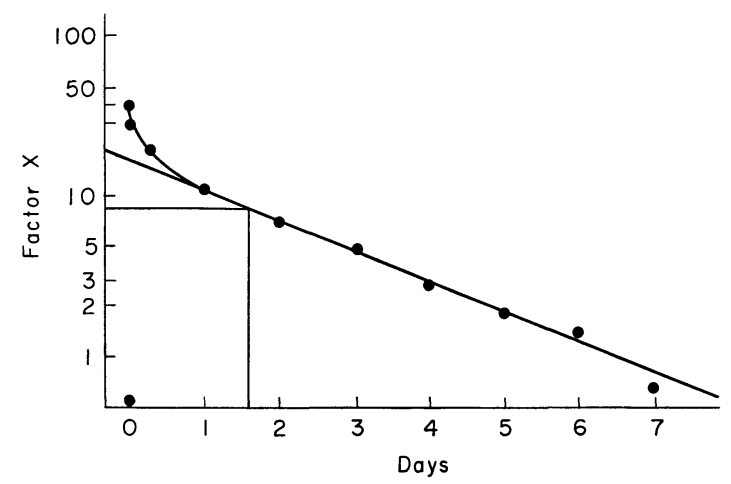

Fig. 2. Disappearance curve of factor $\mathrm{X}$ from plasma after intravenous administration of II, VII, IX and $\mathrm{X}$ concentrate. Case 2.

Case 3 (6-year-old female)

Anamnesis. Birth was normal without excessive hemorrhage. From around the age of 4 , she bled at the nose whenever she caught cold, and subcutaneous hematoma formed easily due to slight injury. From early February, 1975, she complained of pain of the thighs and posterior patellar regions in both legs, the cause of which was unclear. She was hospitalized in Yamagata Prefecture, where anemia and clotting abnormalities were suspected, but without performing definitive diagnosis, blood transfusion was undertaken. In February 1976, she was admitted to our Department for further examination.

Family study. Reduced factor X activity was found in both of her parents (mother $50-68 \%$, father 58 ), but there was no consanguinity among her parents or grandparents and no relatives were found to have hemorrhagic diathesis (Fig. 4).

Physical examinations. Slight anemia of the conjunctiva palpebrae was seen, but no other abnormalities noted.

Laboratory examinations. A RBC count of $4,200,000 / \mathrm{mm}^{3}$ and $\mathrm{Hb}$ value of $63 \%$ indicated mild anemia, but platelet count was normal at $220,000 / \mathrm{mm}^{3}$ and no other abnormalities were seen (Table 1).

Hemostatic examinations. Factor $\mathrm{X}$ activity of this patient was $4.5 \%$, which indicated a relatively mild decrease in comparison with the activity of our other 2 cases $(2.6 \%$ and $1.5 \%$ ). Whole blood clotting time was normal $(10.5 \mathrm{~min})$. K-PTT, however, was markedly prolonged (122.2 sec), the $r$ value $32 \mathrm{~mm}$, indicating mild prolongation (Table 6). Chromosome studies and cross-matching studies with blood from patients with known clotting abnormalities were not performed. 

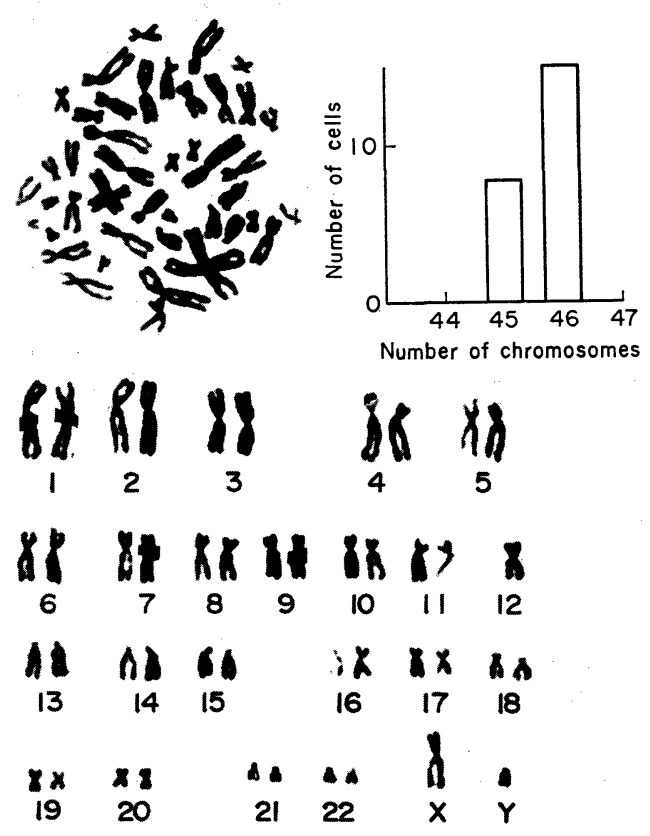

Fig. 3. Karyotype and chromosome number distribution of a factor $\mathrm{X}$ deficiency patient (Case 2).

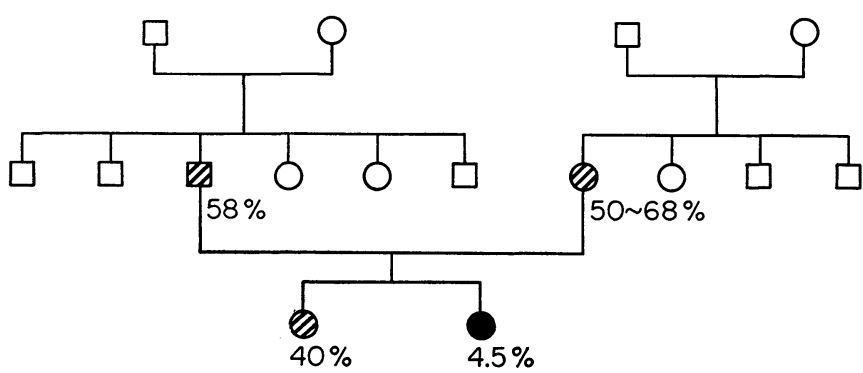

Fig. 4. Pedigree of Case 3.

Effects of intravenous administration of factor $X$ concentrate. Infusion of 2 vials of PPSBNichiyaku (Nihon Pharmaceuticals) produced a biphasic curve, as in the 2 previous cases. The half-life was $56 \mathrm{hr}$ (Fig. 5).

Immunological test for factor $X$. Factor X-like antigen was measured using radioimmunoassay (RIA) and anti-factor $\mathrm{X}$ antiserum in this case and Case 2. Both showed marked reductions $(0.47 \mu \mathrm{g} / \mathrm{ml}$ in Case 2 and $0.15 \mu \mathrm{g} / \mathrm{ml}$ in the present case) in parallel with the reduced factor $\mathrm{X}$ activity, as compared with control values of $15-16 \mu \mathrm{g} / \mathrm{ml}$ (Table 4). 


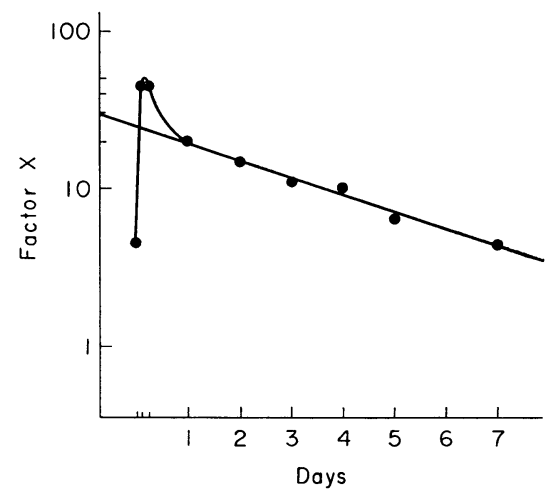

Fig. 5. Disappearance curve of factor $\mathrm{X}$ from plasma after intravenous administration of II, VII, IX and $\mathrm{X}$ concentrate. Case 3 .

TABLE 4. Immunological studies on factor $X$

\begin{tabular}{cccc} 
Normal control & $\begin{array}{c}\text { Factor X } \\
(\mu \mathrm{g} / \mathrm{ml})\end{array}$ & Patients & $\begin{array}{c}\text { Factor X } \\
(\mu \mathrm{g} / \mathrm{ml})\end{array}$ \\
\hline F.K. & 14.15 & M.M. & \\
S.S. & 16.23 & S.U. & 0.47 \\
M.K. & 14.07 & M.K. & 0.15 \\
H.F. & 19.22 & & \\
H.S. & 18.95 & & \\
\hline
\end{tabular}

\section{Review of the Five Japanese Cases}

Clinical symptoms. Clinical data are summarized in Table 5. Including our 3 cases, 5 cases of factor $\mathrm{X}$ deficiency have been reported in Japan. At the time of diagnosis, ages ranged from 6 to 18 years; there were 3 males and 2 females. Positive family histories were found in 3 of the 5 families concerned, all 3 of which showed autosomal recessive characteristics. Consanguinity of parents or grandparents was found in 2 of the 5 cases. The age at the time of first symptoms ranged from 0 to 4 years, among which subcutaneous hemorrhage (some of which formed hematomas) was the most frequent. Intramuscular hemorrhage, hematuria, and umbilical hemorrhage at birth were seen in one case each. Symptoms of subcutaneous and intramuscular hemorrhage prior to examination were found most frequently ( 4 of the 5 cases), followed by epistaxis ( 3 cases), hemarthrosis, gingiva bleeding, digestive tract bleeding, hematuria, bleeding of the conjunctiva, hemoptysis, and retinal hemorrhage in one case each. Petechial hemorrhage etc., as seen in cases of platelet abnormalities, were however not seen.

Hemostatic examinations. Table 6 summarizes the results. Bleeding time in our Case 2 was slightly prolonged to $10 \mathrm{~min}$, but no other cases showed abnormalities. Whole blood clotting time was normal in the case reported by Yasunaga et al. (1964) and in our Case 3 (10.5 min), but the other 3 cases showed mild prolongation (13-20 $\mathrm{min})$. The $r$ value of TEG ranged from $32-64.5 \mathrm{~min}$, indicating mild to moderate prolongation. In contrast, PTT was markedly 
TaBle 5. Clinical data of congenital factor $X$ deficiency in Japan

\begin{tabular}{|c|c|c|c|c|c|}
\hline $\begin{array}{l}\text { Age (years), sex: } \\
\text { Reporter: }\end{array}$ & $\begin{array}{c}\text { Case } 1 \\
8 \text { years old, F } \\
\text { (Yasunaga et } \\
\text { al. 1964) }\end{array}$ & $\begin{array}{c}\text { Case } 2 \\
17 \text { years old, } \\
\text { M (Shirakawa } \\
\text { et al. 1969) }\end{array}$ & $\begin{array}{c}\text { Case } 3 \\
18 \text { years old, } \\
\text { M (Mori et } \\
\text { al. 1972) }\end{array}$ & $\begin{array}{c}\text { Case } 4 \\
11 \text { years old, } \\
\text { M (Mori et } \\
\text { al. 1975a) }\end{array}$ & $\begin{array}{c}\text { Case } 5 \\
6 \text { years old, } \\
\text { F (Mori et } \\
\text { al. } 1976 \text { ) }\end{array}$ \\
\hline Heredity & + & - & + & - & + \\
\hline Consanguinity & + & + & - & - & - \\
\hline Age at onset (years) & 2 & 3 & 4 & 0 & 4 \\
\hline Initial symptom & $\begin{array}{l}\text { Subcutaneous, } \\
\text { Intramuscular }\end{array}$ & Hematuria & $\begin{array}{l}\text { Subcutaneous } \\
\text { hematoma }\end{array}$ & Umbilical & Subcutaneous \\
\hline \multicolumn{6}{|l|}{ Bleeding episode } \\
\hline Subcutaneous & + & - & + & + & + \\
\hline Intramuscular & + & + & + & + & - \\
\hline Haemarthrosis & - & - & - & + & - \\
\hline Epistaxis & - & - & + & + & + \\
\hline Gingival & + & - & - & - & - \\
\hline Gastrointestinal & - & - & - & + & - \\
\hline Hemoptysis & - & - & - & + & - \\
\hline Hematuria & - & + & - & - & - \\
\hline Conjunctival & + & - & - & - & - \\
\hline Retinal & + & - & - & - & - \\
\hline Others & & & & Umbilical* & \\
\hline Blood transfusion & + & - & + & + & - \\
\hline Delivery & & Normal & Normal & Umbilical & Normal \\
\hline
\end{tabular}

* The bleeding lasted for 6 months.

prolonged. In the case reported by Shirakawa et al. (1969), it was $339.3 \mathrm{sec}$ (inactivated PTT) and between 89.1 and 122.2 sec (activated PTT) in our 3 cases. TGT indicated abnormal serum activity with marked prolongation (hypofunction) to $20.6-22.2$ sec in our 3 cases. $\mathrm{RBC}$ count was normal in all cases, as was fibrinolytic activity. Circulating anticoagulant was not apparent. With regard to factor $\mathrm{X}$ activity, our Case 3 had the slightest decrease in function $(4.5 \%)$, followed by our Case 1 at $2.6 \%$. Whole blood clotting time corresponded with such decreases, it being $10.5 \mathrm{~min}$ and $15.5 \mathrm{~min}$, respectively, or in other words normal and near the lower limits of the normal range. The half life of infused factor $\mathrm{X}$ concentrate was between 26.5 and $48 \mathrm{hr}$ in the case reported by Yasunaga et al. (Okamoto et al. 1967) and greater than $24 \mathrm{hr}, 38.6 \mathrm{hr}$ and $56 \mathrm{hr}$ in our 3 cases, each of which was determined once. Although the conditions for the determination were not identical in all cases, it can be said that the half-life of infused factor $\mathrm{X}$ was at least greater than $24 \mathrm{hr}$, between 30 and $60 \mathrm{hr}$. Although some variation was seen with regard to the activity of other clotting factors, no notable abnormalities were observed.

As shown in Table 7, cross-matching tests between the case reported by Yasunaga et al. and our Cases 1 and 2 showed deficiency of the same factor.

Immunological tests were carried out in only our Case 2 and Case 3 , but from cross-matching tests, etc., it is thought that all of the Japanese cases reported thus far have been the classical type. 
TABLE 6. Hemostatic examinations of congenital factor $X$ deficiency in Japan

\begin{tabular}{|c|c|c|c|c|c|}
\hline & \multicolumn{5}{|c|}{ Case reported by } \\
\hline & $\begin{array}{c}\text { Yasunaga et al. } \\
(1964)\end{array}$ & $\begin{array}{c}\text { Shirakawa et al. } \\
(1969)\end{array}$ & $\begin{array}{l}\text { Mori et al. } \\
\text { (1972) }\end{array}$ & $\begin{array}{l}\text { Mori et al. } \\
(1975 a)\end{array}$ & $\begin{array}{l}\text { Mori et al. } \\
\quad(1976)\end{array}$ \\
\hline Bleeding time (min) & Normal & 3.0 & 5.5 & 10.0 & \\
\hline Clotting time ( $\min )$ & Normal & 13 & 15.5 & 20 & 10.5 \\
\hline Kaolin PTT (sec) & Prolonged & $339.3^{*}$ & 89.1 & 106.5 & 122.2 \\
\hline Prothrombin time $(\%)$ & $\begin{array}{l}\text { Remarkably } \\
\text { prolonged }\end{array}$ & 5 & $<10$ & $<10$ & $<10$ \\
\hline Thrombotest (\%) & & 5 & $<5$ & $<5$ & 5.2 \\
\hline \multirow[t]{3}{*}{ Thrombelastgram (mm) } & Prolonged & 33.0 & 53.5 & 64.5 & 32.0 \\
\hline & Normal & 8 & 20.5 & 22.5 & 13.5 \\
\hline & Normal & 67 & 44.0 & 53.8 & 52.0 \\
\hline $\begin{array}{l}\text { Thromboplastin screening test } \\
\text { Thromboplastin generation test }\end{array}$ & Prolonged & Abnormal & & & \\
\hline $\begin{array}{l}\text { Thromboplastin generation test } \\
\text { (sec) } \\
\begin{array}{cl}\text { plasma } \\
\text { serum } \\
\text { platelet }\end{array}\end{array}$ & $\begin{array}{l}\text { Normal } \\
\text { Abnormal } \\
\text { Normal }\end{array}$ & Abnormal & $\begin{array}{r}8.0 \\
22.2 \\
10.1\end{array}$ & $\begin{array}{r}9.4 \\
20.6\end{array}$ & $\begin{array}{r}8.6 \\
21.2\end{array}$ \\
\hline \multirow{5}{*}{$\begin{array}{l}\text { Platelet count }\left(\times 10^{4} / \mathrm{mm}^{3}\right) \\
\text { Factor X (\%) } \\
\text { Half life of factor X }(\mathrm{hr}) \\
\text { Fibrinolysis } \\
\text { Circulating anticoagulant } \\
\text { Other clotting factors }\end{array}$} & Normal & 38.2 & 24.0 & 30.1 & 22.0 \\
\hline & 1 & 0.1 & 2.6 & 1.5 & 4.5 \\
\hline & $26.5-48$ & & $>24$ & 38.6 & 56 \\
\hline & $\begin{array}{r}\text { Normal } \\
(-)\end{array}$ & & Normal & $\begin{array}{l}\text { Normal } \\
(-)\end{array}$ & Normal \\
\hline & $\begin{array}{l}\text { II, V : normal } \\
\text { VII complex: } \\
\text { decreased }\end{array}$ & $\begin{array}{l}\text { C.R.: normal } \\
\text { I: } 381 \mathrm{mg} / 100 \mathrm{ml} \\
\text { II: } 95 \% \\
\text { V: } 30 \% \\
\text { VII: } 85 \% \\
\text { VII complex: } 0.1 \%\end{array}$ & $\begin{array}{l}\text { I: } 156.7 \mathrm{mg} / 100 \mathrm{ml} \\
\text { II }: 96 \% \\
\text { V: } 100 \% \\
\text { VII: } 52 \% \\
\text { VIII: } 120 \% \\
\text { IX: } 96 \% \\
\text { XIII: normal }\end{array}$ & $\begin{array}{l}\text { I: } 485.3 \mathrm{mg} / 100 \mathrm{ml} \\
\text { II: } 100 \% \\
\text { V: } 100 \% \\
\text { VII: } 100 \% \\
\text { VIII }: 74 \% \\
\text { IX: } 55 \% \\
\text { XI: } 100 \% \\
\text { XII: } 100 \% \\
\text { XIII: normal }\end{array}$ & $\begin{array}{l}\text { I: } 240 \mathrm{mg} / 100 \mathrm{ml} \\
\text { II: } 82 \% \\
\text { V: } 76 \% \\
\text { VII }: 72 \%\end{array}$ \\
\hline
\end{tabular}

* Standard PTT. 


\section{Discussion}

Factor X deficiency was first reported by Telfer et al. (1956) as "a 'new' coagulation defect", subsequently known as Prower defect. Until 1978 only 43 such cases (Tables 9-12) were reported in the world literature, indicating that it is an extremely rare congenital hemorrhagic diathesis. Including our 3 cases, 5 cases have been reported in Japan, all of which have been the classical type.

\section{Clinical considerations}

Excluding our 3 cases, a total of only 40 cases have been reported -31 of which were the classical type and 9 of which showed abnormal factor $\mathrm{X}$ disorder. Differences according to locality of race have not been found. Both the classical and the abnormal types are said to be inherited as autosomal recessives, but the records are unclear in many cases.

Lechler et al. (1965) investigated the factor $\mathrm{X}$ activity in 42 family members related to 2 homozygous siblings with factor $\mathrm{X}$ deficiency. He found 3 distinct groups: 1) homozygous normal (normal factor $\mathrm{X}$ activity), 2) heterozygous abnormal (factor $\mathrm{X}$ activity about $50 \%$ ), 3) homozygous abnormal (factor $\mathrm{X}$ activity less than $1 \%$ ). The factor $\mathrm{X}$ activity of these 3 groups were said not to overlap. Therefore, a case with hemorrhagic symptoms was from group 3. From these facts, they maintained that this disease is inherited as an autosomal recessive and that normals and asymptomatic heterozygote individuals (carrier: factor $\mathrm{X}$ activity $40 \%-68 \%$ ) could be distinguished. Genetic counseling was, therefore, recommended for members of such families - both families with factor $\mathrm{X}$ deficiency

TaBLE 7. Cross-machting tests between the case reported by Yasunaga et al. and our Case 1 and Case 2

\begin{tabular}{|c|c|c|c|c|c|c|}
\hline $\begin{array}{l}\text { Normal } \\
\text { plasma } \\
(\mathrm{ml})\end{array}$ & $\begin{array}{c}\text { Plasma from } \\
\text { the case } \\
\text { reported by } \\
\text { Yasunaga et al. } \\
(1964) \\
(\mathrm{ml})\end{array}$ & $\begin{array}{l}\text { Case } 1 \\
\text { plasma } \\
\\
(\mathrm{ml})\end{array}$ & $\begin{array}{c}\text { Case } 2 \\
\text { plasma } \\
(\mathrm{ml})\end{array}$ & $\begin{array}{l}\text { Saline } \\
(\mathrm{ml})\end{array}$ & $\begin{array}{c}\text { Prothrombin } \\
\text { time } \\
\text { (sec) }\end{array}$ & $\begin{array}{c}\text { Corrective } \\
\text { effect }\end{array}$ \\
\hline \multirow[t]{4}{*}{0.09} & & & & 0.01 & 13.7 & \\
\hline & 0.09 & & & 0.01 & 52.7 & \\
\hline & & 0.09 & & 0.01 & 200 & \\
\hline & & & 0.09 & 0.01 & 118.2 & \\
\hline 0.09 & 0.01 & & & & 12.9 & + \\
\hline 0.01 & 0.09 & & & & 18.5 & + \\
\hline 0.09 & & 0.01 & & & 12.1 & + \\
\hline 0.01 & & 0.09 & & & 200 & - \\
\hline 0.09 & & & 0.01 & & 13.0 & + \\
\hline \multirow[t]{7}{*}{0.01} & & & 0.09 & & 21.6 & + \\
\hline & 0.09 & 0.01 & & & 58.4 & - \\
\hline & 0.01 & 0.09 & & & 200 & - \\
\hline & 0.09 & & 0.01 & & 47.4 & - \\
\hline & 0.01 & & 0.09 & & 91.4 & - \\
\hline & & 0.09 & 0.01 & & 200 & - \\
\hline & & 0.01 & 0.09 & & 120 & - \\
\hline
\end{tabular}


TABLE 8. Classical factor $X$ deficiency in the world literature

\begin{tabular}{|c|c|c|c|c|c|c|c|}
\hline Case & Reporter & Age & Sex & $\begin{array}{l}\text { Age at } \\
\text { onset }\end{array}$ & $\begin{array}{c}\text { Initial } \\
\text { symptoms }\end{array}$ & $\begin{array}{l}\text { Consangui- } \\
\text { nity }\end{array}$ & $\begin{array}{l}\text { Blood } \\
\text { transfusion }\end{array}$ \\
\hline 1 & Telfer et al. (1956) & $22 \mathrm{yr}$. & $\mathrm{F}$ & $5 \mathrm{yr}$. & $\begin{array}{l}\text { Tooth } \\
\text { extraction }\end{array}$ & - & + \\
\hline 2 & Hougie et al. (1957) & $36 \mathrm{yr}$. & M & & & & + \\
\hline 3 & Bachman et al. (1957) & $1 \mathrm{yr}$ & $\mathbf{F}$ & $0 \mathrm{yr}$. & Melena & & W \\
\hline 4 & Lorraince et al. (1958) & & $\mathrm{F}$ & & & & \\
\hline 5 & Oehme et al. (1958) & $1 \mathrm{yr}$. & & & & & \\
\hline 6 & Caen et al. (1958) & $6 \mathrm{yr}$. & $\mathrm{M}$ & & & & + \\
\hline 7 & Roos et al. (1959) & & & & & & \\
\hline 8 & Hoerder (1959) & $27 \mathrm{yr}$. & $\mathrm{F}$ & $14 \mathrm{yr}$. & & + & W \\
\hline 9 & Brody \& Stuart (1960) & $27 \mathrm{yr}$. & $\mathrm{F}$ & $5 \mathrm{yr}$. & Tonsillectomy & & + \\
\hline 10 & $\begin{array}{c}\text { Rabiner \& Kretchmer } \\
(1961)\end{array}$ & $6 \mathrm{yr}$. & $\mathrm{M}$ & $1 \mathrm{yr}$. & Gingival & & \\
\hline 11 & Ascari et al. (1964) & $42 \mathrm{yr}$. & M & & & & \\
\hline 12 & Pigeaud et al. (1964) & $18 \mathrm{yr}$. & & & & & \\
\hline 13 & Yasunaga et al. (1964) & $8 \mathrm{yr}$. & $\mathrm{F}$ & $2 \mathrm{yr}$. & Subcutaneous & + & H \\
\hline 14 & Barkazan et al. (1965) & $54 \mathrm{yr}$. & $\mathbf{F}$ & $5 \mathrm{yr}$. & & & \\
\hline 15 & Lechler et al. (1965) & $13 \mathrm{yr}$. & $\mathrm{F}$ & $4 \mathrm{yr}$. & Subcutaneous & & + \\
\hline 16 & " & $4 \mathrm{yr}$. & $\mathrm{M}$ & & & & + \\
\hline 17 & Holzknecht (1965) & $20 \mathrm{yr}$. & $\mathrm{M}$ & & & & + \\
\hline 18 & Shirakawa et al. (1969) & $17 \mathrm{yr}$. & $\mathrm{M}$ & 3 yr. & Hematuria & + & \\
\hline 19 & Szönyi et al. (1969) & $11 \mathrm{yr}$. & $\mathrm{F}$ & & & & \\
\hline 20 & Kurz et al. (1969) & $9 \mathrm{yr}$. & $\mathrm{M}$ & $6 \mathrm{yr}$. & Subcutaneous & & \\
\hline 21 & " & $8 \mathrm{yr}$. & $\mathrm{M}$ & $1 \mathrm{mo}$. & & & \\
\hline 22 & " & $1 \mathrm{mo}$. & $\mathrm{F}$ & & Umbilical & & \\
\hline 23 & Girolami et al. (1970) & $5 \mathrm{mo}$. & M & $0 \mathrm{mo.}$ & Melena & - & \\
\hline 24 & Sarup et al. (1972) & & M & & & + & \\
\hline 25 & I " & $7 \mathrm{mo}$ & M & & & - & + \\
\hline 26 & Gonzalo et al. (1973) & $21 \mathrm{yr}$. & $\mathrm{F}$ & $8 \mathrm{yr}$. & & & \\
\hline 27 & " & $19 \mathrm{yr}$. & $\mathbf{F}$ & $8 \mathrm{yr}$. & & & \\
\hline 28 & " & $16 \mathrm{yr}$. & $\mathrm{M}$ & $6 \mathrm{yr}$. & & & \\
\hline 29 & " & $12 \mathrm{yr}$. & $\mathrm{F}$ & $2 \mathrm{yr}$. & & & \\
\hline 30 & Girolami et al. (1975) & $28 \mathrm{yr}$ & $\mathrm{M}$ & & & - & \\
\hline 31 & Schneegans et al. (1976) & & & 0 mo. & & & + \\
\hline
\end{tabular}

and families with partial clotting function deficiency inherited as autosomal recessives, other than hemophilia. Various hemorrhagic symptoms due to clotting abnormalities were found (Tables 9 and 11).

\section{Hemostatic considerations}

Prolongation of whole blood clotting time, one stage prothrombin time, partial thromboplastin time and the $r$ and $k$ values of TEG were seen and reduced factor X activity found (Table 10). The fact that the one stage PT was prolonged even using RVV indicates that it is classical factor $\mathrm{X}$ deficiency, that is, an absolute deficiency of factor $\mathrm{X}$, ranging from 0.1 to $14 \%$. Cases showing normal or nearly normal values were diagnosed as abnormal factor $\mathrm{X}$ disorder. Although factor $\mathrm{X}$ activity was between $4 \%$ and $14 \%$ as determined by tissue thromboplastin, it was normal, between $60 \%$ and $104 \%$, as determined by RVV. 
TABLe 9. Hemorrhagic symptoms of classical factor $X$ deficiency

\begin{tabular}{|c|c|c|c|c|c|c|c|c|c|}
\hline Case & $\begin{array}{l}\text { Subcutaneous } \\
\text { hemorrhage }\end{array}$ & Epistaxis & $\begin{array}{c}\text { Gingival } \\
\text { hemorrhage }\end{array}$ & $\begin{array}{l}\text { Intramuscular } \\
\text { hemorrhage }\end{array}$ & $\begin{array}{l}\text { Intraarticular } \\
\text { hemorrhage }\end{array}$ & $\begin{array}{l}\text { G.I. tract } \\
\text { hemorrhage }\end{array}$ & Hematuria & $\begin{array}{l}\text { Genital } \\
\text { bleeding }\end{array}$ & Others \\
\hline 1 & & & + & & & & & & After tonsillectomy \\
\hline 2 & & H & & H & + & & & & \\
\hline 3 & & & & + & & + & & & \\
\hline 4 & & & & & & & & & \\
\hline 5 & & & & + & + & & & & \\
\hline 6 & + & & & & & + & & & \\
\hline 7 & + & + & + & & + & & & + & \\
\hline 8 & + & & & + & & & & & Ovarian \\
\hline 9 & + & + & + & & & & + & & \\
\hline 10 & + & + & + & & & + & + & & \\
\hline 11 & + & + & + & + & & & & & Tongue \\
\hline 12 & + & & & & + & + & & & \\
\hline 13 & + & & + & + & & & & & Subconjunctival \\
\hline 14 & & & & + & & & & + & Hemoptysis \\
\hline 15 & + & & & & + & & & + & Posttraumatic \\
\hline 16 & + & & & & + & & & + & Posttraumatic \\
\hline 17 & & H & \# & H & \# & & + & & Posttraumatic \\
\hline 18 & + & & & + & + & & & & \\
\hline 19 & & & & & & & & & \\
\hline 20 & H & & & & + & & & & \\
\hline 21 & + & H & + & + & H & & & & \\
\hline 22 & & \# & H & + & \# & & & & Umbilical \\
\hline 23 & & & & + & + & + & & & Cerebral \\
\hline 24 & + & + & & + & + & & & & Tongue \\
\hline 25 & & & + & & & & & & \\
\hline 26 & + & Ht & H & + & + & & & H & \\
\hline 27 & + & H & \# & + & H & & & m & \\
\hline 28 & + & \# & + & H & H & & & & \\
\hline 29 & H & H & + & + & + & & & & \\
\hline 30 & + & + & & + & + & & + & & \\
\hline 31 & + & & & + & & + & & & Cerebromeningeal \\
\hline
\end{tabular}


TaBle 10. Hemostatic examinations of classical factor $X$ deficiency

\begin{tabular}{|c|c|c|c|c|c|}
\hline Case & $\mathrm{CT} / \mathrm{N}$ & $\mathrm{PT} / \mathrm{N}$ & $\mathrm{PTT} / \mathrm{N}$ & Factor X & $\mathrm{RVV}$ time/N \\
\hline 1 & $8^{\prime} 8^{\prime \prime} / 8-10^{\prime}$ & $24-30^{\prime \prime} / 8.8-12^{\prime \prime}$ & & $7 \%$ & $\mathrm{~N}, \mathrm{Abn}$ \\
\hline 2 & $14^{\prime}$ & Abn & 137" Abn & $1-3 \%$ & Abn \\
\hline 3 & & Abn & (RT: Abn) & $1 \%$ & Abn \\
\hline 4 & $23^{\prime} / 10-20^{\prime}$ & $54^{\prime \prime} / 21^{\prime \prime}$ & $94^{\prime \prime} / 27-43^{\prime \prime}$ & & Abn \\
\hline 5 & $11^{\prime}$ & & (RT 125-168"/90-135") & & \\
\hline 6 & & $51^{\prime \prime} / 13^{\prime \prime}$ & & $1 \%$ & $29^{\prime \prime} / 22^{\prime \prime}$ \\
\hline 7 & $10-11^{\prime} / 5-8.5$ & $51^{\prime \prime} / 15^{\prime \prime}$ & $\left(\mathrm{RT} 180^{\prime \prime} / 180^{\prime \prime}\right)$ & $5 \%$ & \\
\hline 8 & $9^{\prime} 40^{\prime \prime} / 5 \cdot 8^{\prime}$ & $9 \%$ & & $1-3 \%$ & \\
\hline 9 & $20^{\prime}$ & $5 \%$ & & & \\
\hline 10 & $\mathrm{Abn}$ & $\mathrm{Abn}$ & & $10 \%$ & \\
\hline 11 & $11-14 / 7-10^{\prime}$ & $36^{\prime \prime} / 13^{\prime \prime}$ & $\left(\mathrm{RT} 190^{\prime \prime} / 110^{\prime \prime}\right)$ & $2 \%$ & \\
\hline 12 & & & & $10 \%$ & \\
\hline 13 & & Abn & Abn (RT: Abn) & $1.0 \%$ & \\
\hline 14 & & $14-38 \%$ & & & $(\mathrm{FII}, \mathrm{V}, \mathrm{VII}: \mathrm{N})$ \\
\hline 15 & Abn & Abn & Abn & $1.0 \%$ & \\
\hline 16 & Abn & Abn & Abn & $1.0 \%$ & \\
\hline 17 & & $10 \%$ & & $10 \%$ & \\
\hline 18 & $13^{\prime}$ & $5 \%$ & $339^{\prime \prime} / 40-100^{\prime \prime}$ & $0.1 \%$ & Abn \\
\hline 19 & $11-15^{\prime}$ & $39-44^{\prime \prime} / 15-17^{\prime \prime}$ & $110-130^{\prime \prime} / 55-80^{\prime \prime}$ & $(\mathrm{FX}+\mathrm{VII}: 19 \%)$ & \\
\hline 20 & & $17 \%$ & & $14 \%$ & \\
\hline 21 & & $13 \%$ & & $10 \%$ & \\
\hline 22 & & $10 \%$ & & $10 \%$ & \\
\hline 23 & $20^{\prime}$ & $111^{\prime \prime} / 13^{\prime \prime}$ & $240^{\prime \prime} / 45^{\prime \prime}$ & $0.1 \%$ & \\
\hline 24 & $11^{\prime} 25^{\prime \prime}$ & $45^{\prime \prime} / 12-14^{\prime \prime}$ & $102^{\prime \prime} / 30-50^{\prime \prime}$ & & $58^{\prime \prime} / 17-20^{\prime \prime}$ \\
\hline 25 & $40^{\prime}$ & $96^{\prime \prime} / 12-14^{\prime \prime}$ & $76^{\prime \prime} / 30-50^{\prime \prime}$ & & $120^{\prime \prime} / 17-20^{\prime \prime}$ \\
\hline 26 & $6^{\prime}$ & $23^{\prime \prime} / 11-13^{\prime \prime}$ & $95^{\prime \prime} / 60^{\prime \prime}$ & $5 \%$ & $35^{\prime \prime} / 10-15^{\prime \prime}$ \\
\hline 27 & $6^{\prime} 30^{\prime \prime}$ & $21^{\prime \prime} / 11-13^{\prime \prime}$ & $100^{\prime \prime} / 60^{\prime \prime}$ & $5 \%$ & $26^{\prime \prime} / 10-15^{\prime \prime}$ \\
\hline 28 & $8^{\prime}$ & $27^{\prime \prime} / 11-13^{\prime \prime}$ & $93^{\prime \prime} / 60^{\prime \prime}$ & $5 \%$ & $30^{\prime \prime} / 10-15^{\prime \prime}$ \\
\hline 29 & $12^{\prime}$ & $22^{\prime \prime} / 11-13^{\prime \prime}$ & $98^{\prime \prime} / 60^{\prime \prime}$ & $5 \%$ & $32^{\prime \prime} / 10-15^{\prime \prime}$ \\
\hline 30 & & & & $1 \%$ & \\
\hline 31 & & $120^{\prime \prime} / 11-11.5^{\prime \prime}$ & & $1 \%$ & $80^{\prime \prime} / 13.2-13.7^{\prime \prime}$ \\
\hline
\end{tabular}

Immunological considerations (as regards abnormal factor $X$ )

Girolami et al. (1970) made an investigation of one female (67 years old) and 2 males (40 and 46 years old) suspected of having factor $\mathrm{X}$ deficiency. Clotting studies using tissue and partial thromboplastin showed factor $\mathrm{X}$ activity of $4 \%$ to $9 \%$, but using a Stypven-cephalin mixture, normal values were obtained. They hypothesized, therefore, that rather than an actual deficiency, these paticnts had abnormal factor $\mathrm{X}$. This hypothesis was supported by antibody neutralization tests and immunodiffusion tests. That is, the activation of abnormal factor $\mathrm{X}$ using tissue or partial thromboplastin was reduced, but normal using RVV - indicating the presence of a factor somewhat different from normal factor $\mathrm{X}$. Borrowing the name of the location in northeastern Italy where these cases were found, they have named this disease "Factor X Friuli".

Similar to classical factor $\mathrm{X}$ deficiency, the factor $\mathrm{X}$ activity of heterozygotes (carriers) is about $50 \%$, and hemorrhagic symptoms, such as major hemorrhage due to trauma or hemorrhage after tooth extraction, are often found.

Genetically, it is also thought to be transferred as an autosomal recessive. Denson (1958) made a cross-matching test of the plasma from cases of 
TABLE 11. Abnormal factor $X$

\begin{tabular}{|c|c|c|c|c|c|c|c|c|c|c|c|}
\hline Case & \multicolumn{2}{|c|}{ Reporter } & 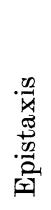 & 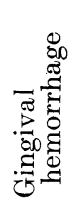 & 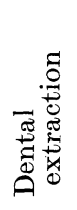 & 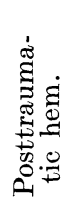 & 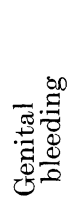 & 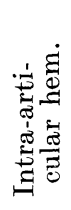 & 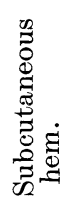 & 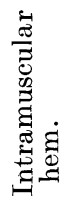 & 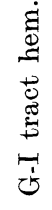 \\
\hline 1 & \multicolumn{2}{|c|}{ Girolami et al. (1969) } & + & + & + & + & + & & & & \\
\hline 2 & \multicolumn{2}{|c|}{ " } & + & + & + & & & + & & & \\
\hline 3 & \multicolumn{2}{|l|}{$"$} & + & + & + & & & & & & \\
\hline 4 & \multirow{2}{*}{\multicolumn{2}{|c|}{$\begin{array}{l}\text { " } \\
\text { " }\end{array}$}} & + & + & + & & & & & & \\
\hline 5 & & & + & + & + & + & & & & & \\
\hline 6 & $"$ & (1970) & + & & + & + & & - & & + & \\
\hline 7 & $"$ & (1971) & + & & + & & $H$ & + & + & + & + \\
\hline 8 & $"$ & (1973) & + & & $H$ & + & + & - & + & + & \\
\hline 9 & Parkin et al. ( & 1974) & & & & & & & & & \\
\hline
\end{tabular}

Prower defect and Stuart defect and found no correction, leading them to conclude that they are due to the same factor defect. Furthermore, they maintain that the report of Telfer et al. (1956) using RVV (which came to contradictory conclusions) is incorrect due to the technical errors.

\section{Diagnosis}

Patients with hemorrhagic symptoms characteristic of clotting factor deficiencies from childhood should be suspected of clotting abnormalities, and deñnitive diagnosis be made by clotting studies. Immunological tests are also necessary.

\section{Therapy}

Replacement therapy. Blood transfusion has long been used as the sole effective means for hemostasis in hemophilia. Together with the development in blood coagulation studies, however, it has become possible to provide therapy by injection of purified clotting factors rather than whole blood transfusion. Macfarlane et al. (1954) succeeded in controlling hemophilic hemorrhage following the isolation of anti-hemophilic globulin ( $\mathrm{AHG}$ ) from the blood of experimental animals. Since, however, various side-effects arise due to its antigenicity when applied to man, AHG was then isolated from human subjects (Kekwick and Wolf 1957), and today plays an important clinical role in controlling hemophilic hemorrhage.

Advances with regard to hemophilia B have proceeded somewhat slower. From 1960 to 1962, such a concentrate was developed and put to clinical use in Japan in 1970. The effective concentrates for hemophilia B include PSSB-Nichiyaku (Nihon Pharmaceuticals, Takeda Pharmaceuticals, Japan: P, prothrombin; P, proconvertin; S, Stuart; B, Hemophilia B), Konyne (Cutter Laboratories, USA), 
disorders in the world literature

\begin{tabular}{|c|c|c|c|c|c|c|}
\hline \multirow{2}{*}{$\underset{\text { (years) }}{\text { Age }}$} & \multirow{2}{*}{ Sex } & \multirow{2}{*}{$\mathrm{PT}$ (sec) } & \multirow{2}{*}{ PTT (sec) } & \multicolumn{2}{|c|}{ Factor X } & \multirow{2}{*}{ TEG $(\mathrm{mm})$} \\
\hline & & & & T. TP (\%) & $\operatorname{RVV}(\%)$ & \\
\hline 67 & $\mathrm{~F}$ & 33.7 & 66.6 & 7 & 92 & \\
\hline 40 & $\mathrm{M}$ & 29.3 & 67.7 & 8 & 76 & $\begin{array}{l}\mathrm{r}=42 \\
\mathrm{k}=21\end{array}$ \\
\hline 8 & $\mathrm{M}$ & 33.2 & 80.7 & & & \\
\hline 25 & M & 23.8 & 50.4 & & & \\
\hline 23 & $\mathrm{M}$ & $\begin{array}{c}31.0 \\
(N=13-15)\end{array}$ & $\begin{array}{c}81.5 \\
(N<45)\end{array}$ & & & \\
\hline 46 & M & & & 4 & 70 & \\
\hline 43 & $\mathrm{~F}$ & 33.3 & 66.7 & 9 & 60 & $\begin{array}{l}\mathrm{r}=72 \\
\mathrm{k}=35\end{array}$ \\
\hline 32 & F & $\begin{array}{c}33.5 \\
(N=13)\end{array}$ & $\begin{array}{c}72.5 \\
(N=45)\end{array}$ & 14 & 60 & $\begin{aligned} \mathrm{r} & =34 \\
\mathrm{k} & =22\end{aligned}$ \\
\hline 52 & $\mathrm{M}$ & $\begin{array}{c}12.0 \\
(N=16)\end{array}$ & $\begin{array}{c}50.0 \\
(N=32-40)\end{array}$ & 8.5 & 105 & \\
\hline
\end{tabular}

Christmassin (Green Cross, Japan), etc. Since other than factor IX, factors II, VII and $\mathrm{X}$ are also included, as seen in fractionation studies, they are effective for deficiencies of each of these other factors (Mori et al. 1975b). Such therapy for hemophilia and similar diseases has come to be called "replacement therapy", and appears to be almost totally effective. As seen from the results of our cases as well, use of primarily PPSB-Nichiyaku at the time of hemorrhage can be recommended.

With regard to the half-life of factor $\mathrm{X}$ administered intravenously with plasma, serum or concentrates, various reports have been made. Biggs and Denson (1963) reported a half-life of $48 \mathrm{hr}$, Roberts et al. (1965) 20-24 hr, Okamoto et al. (1967) 26.5-48 hr, Kurz et al. (1969) 48 hr, Rizza (1972) 2-3 days, and Bidwell et al. (1972) 60-70 hr, etc. The half-life for our three cases (greater than $24 \mathrm{hr}, 38.6 \mathrm{hr}$, and $56 \mathrm{hr}$ ) agrees generally with those of previous reports. In the 2 cases of abnormal factor X disorder reported by Girolami et al. (1974), the in vivo half-life was 30 and $28 \mathrm{hr}$. They maintain that, as in the abnormal factor IX in hemophilia, there is no inhibitory activity in abnormal factor $\mathrm{X}$ disorders.

Rizza (1972) reported a hemostatic level greater than $10 \%$, Bidwell et al. (1972) a level between 10 and 40\%, Okamoto et al. (1967) 15-20\% and Sarup et al. (1972) $15 \%$. In abnormal factor X disorders, Girolami et al. (1974) maintain that $45-50 \%$ is sufficient for hemostasis at the time of hematoma formation due to tooth extraction, hematuria, trauma, etc. With regard to the hemostatic level at the time of major surgery, Krieger et al. (1977) have reported that in all diseases of coagulation abnormalities, the hemostatic level is $100 \%$ prior to surgery, greater than $60 \% 4$ days postoperatively, and greater than $40 \% 8$ days postoperatively. They maintain that it is necessary to keep a level greater than $40 \%$ until removal of sutures and that daily monitoring of clotting factors is required. At the 
time of our surgery case for a broken right femur at the cervical region, factor $\mathrm{X}$ activity during the operation was $100 \%$ due to administration of factor $\mathrm{X}$ concentrate. Three days postoperatively it was greater than $50 \%$. It was maintained at greater than $30 \%$ for 2 weeks and $10-20 \%$ for 3 months during functional training. Later hemorrhage did not appear and the postoperative course was favorable (Sakai et al. 1979).

During the early years using replacement therapy, whole blood and whole plasma were used and it was just possible to maintain only a minimum level of hemostasis by technical means. More recently, with the development, improvement and widespread usage of concentrates, generally high levels of factor $\mathrm{X}$ have been administered for hemostasis. In either case, it is known that the in vivo halflife of factor $\mathrm{X}$ is relatively long, so that effective hemostasis can be achieved by replacement therapy. Therapy has consequently become relatively easy.

Hormone therapy with progesterone derivatives. Brody and Stuart (1960) reported a case of a 27-year-old woman with factor $\mathrm{X}$ deficiency. She had experienced epistaxis, gingiva bleeding, hematuria, genital bleeding and repeated subcutaneous hematomas due to mild injuries since infancy. Major hemorrhage occurred at the time of adenoid removal. From the third month of pregnancy, all symptoms disappeared. Six weeks later, the formerly prolonged 1 stage prothrombin time $(170 \mathrm{sec})$ had shortened markedly to $22 \mathrm{sec}$. Such clinical and hematological improvement continued until parturition. They speculated that there is some correlation between the endocrine system and certain clotting factors.

The same patient was treated 4 years later in 1964 (at the age of 31) by Haber (1964) with the progesterone derivative, norethynodrel (Ernovid, $10 \mathrm{mg} / \mathrm{day}$ ), and improvement found. That is, after the birth of her third child, hemorrhagic symptoms reappeared, and one week following the start of treatment with norethynodrel, prothrombin time had shortened from $800 \mathrm{sec}$ to $17 \mathrm{sec}$ and all symptoms disappeared. Since reduction to a dose of $5 \mathrm{mg} /$ day resulted in reappearance of hemorrhage, $10 \mathrm{mg} /$ day was thought as appropriate. Such treatment continued for over one year with maintenance of PT at 16-18 sec and with no symptoms returning. The changes due to administration of this progesterone derivative were identical to those seen during pregnancy. It is of course known that a hypercoagulable state is present during pregnancy due to the increase in Fbg, and factors II, VII, IX and X. It is thought that this is due to the direct effect of hormones from the placenta on the synthesis of factor X. Endogenous progesterones markedly increase during pregnancy due to development of placenta and ovulation or maturation of ovarian follicles is prevented due to interference with pituitary gonadotrophin secretion. Subsequently, the secondary physical changes similar to those in pregnancy - amenorrhea, morning sickness, stimulation of breast tissue growth, etc. - occur. Administration of progesterone derivatives in this patient demonstrates the fact that a hypercoagulable state is also produced together with the change of pregnancy. Specifically, from clinical and coagulation studies, it is concluded that the effects on factor $\mathrm{X}$ deficiency caused by pregnancy 
or norethynodrel are due to excessive progesterone or norethynodrel, which produces increased factor $\mathrm{X}$ production, rather than acting as a substitute for factor X. Nonetheless, controversy still remains with regard to this point (Johnson 1957; Oehme et al. 1958; McGovern et al. 1961).

Because of concern regarding possible clotting and hormonal side-effects, we have not performed hormonal therapy in our three cases, but we believe that it is a method worth trying in cases of fully matured women.

Other therapy. Various hemostatics were administered alone or in combination with the above methods, but judging from the fact that the activation of abnormal factor $\mathrm{X}$ was about normal, as determined by RVV, it is expected that various snake venoms should be effective in cases of abnormal factor $\mathrm{X}$ disorders.

\section{Conclusion}

Having experienced 3 cases of congenital factor $\mathrm{X}$ deficiency, which is a rare disease among the clotting factor deficiencies, we have reported the clinical findings, results of coagulation studies, therapy and an immunological investigation of factor $\mathrm{X}$.

1. The cases were an 18-year-old male, 11-year-old male, and a 6-year-old female - which were, respectively, the third, fourth and fifth cases reported in Japan. Consanguinity was not found in any case. Umbilical bleeding, and subcustaneous and intramuscular hemorrhage were experienced from infancy through childhood, as well as a variety of hemorrhagic symptoms.

2. Coagulation studies revealed: a) mild prolongation of whole blood clotting time and $r$ and $k$ values of TEG; b) marked prolongation of PT and PTT; c) reduced serum activity, as determined by TGT; and d) marked reduction of PT, PTT and factor $\mathrm{X}$ activity as seen using both tissue thromboplastin and RVV. e) The in vivo factor $\mathrm{X}$ half-life following administration of factor $\mathrm{X}$ concentrate was found to be $24,38.6$ and $56 \mathrm{hr}$ in these 3 cases.

3. Immunological study of factor $\mathrm{X}$ showed extremely low levels of factor $\mathrm{X}$ like antigen in our second and third cases $(0.47 \mu \mathrm{g} / \mathrm{ml}$ and $0.15 \mu \mathrm{g} / \mathrm{ml}$, respectively), indicating that the factor $\mathrm{X}$ deficiency was a classical type.

\section{References}

1) Ascari, E., Barbieri, U. \& Gobbi, F. (1964) Deficit congenito del fattore Stuart. Minerva medica, 326, 2873-2878.

2) Bachmann, F., Duckert, F., Flickiger, P. \& Hitzig, H. (1957) Haemorrhagische Diathesen verursacht durch Mangel des Stuart-Prower-Faktors. Schweiz. med. Wschr., 87, 1221-1223.

3) Barkazan, Z.S., Sukhoveena, E. Ya. \& Khmara, L. Ya. (1965) Primary hemorrhagic diathesis associated with deficiency of the Stuart-Prower factor. Clin. Med., 43, 120125.

4) Biggs, R. \& Denson, K.W.E. (1963) The fate of prothrombin and factor VII, IX, X transfused to patients deficient in these factors. Brit. J. Haemat., 9, 532-547.

5) Bidwell, E., Dike, G.W.R. \& Ford, W.H. (1972) Therapeutic materials. In: Human Blood Coagulation, Haemostasis and Thrombosis, edited by R. Biggs, Blackwell Publishing Co., London-Melborne, pp. 225-277. 
6) Brody, J.I. \& Stuart, C.F. (1960) Improvement of factor X deficiency during pregnancy. New Engl. J. Med., 20, 996-999.

7) Caen, J., Beaumont, J.L. \& Bernard, J. (1958) Deficit en Facteur Stuart. Etude biol. Rev. Franc. Etudes. clin. Biol., 3, 161-163.

8) Denson, K.W.E. (1958) Electrophoretic studies of the Prower factor: A blood coagulation factor which differs from factor VII. Brit. J. Haemat., 19, 313-325.

9) Girolami, A., Molaro, G., Lazzarin, M. \& Scarpa, R. (1969) Una nuova coagulopatia emorrhagica congenita probabilmente dovuta alla presenza di un fattore $\mathrm{X}$ abnorme. Minerva medica, 60, 4929-4949.

10) Girolami, A., Molaro, G., Lazzarin, M., Scarpa, R. \& Brunetti, A. (1970) A 'New' congential haemorrhagic condition due to the presence of an abnormal factor $\mathrm{X}$ (Factor X Friuli). Study of a large kindred. Brit. J. Haemat., 19, 179-192.

11) Girolami, A., Lazzarin, M., Scarpa, R. \& Brunetti, A. (1971) Further studies on the abnormal factor $\mathrm{X}$ (Factor $\mathrm{X}$ Friuli) coagulation disorder: A report of another family. Blood, 37, 534-541.

12) Girolami, A., Nicolini, R., Furlani, E. \& Bareggi, G. (1973) Abnormal factor X (Factor X Friuli) coagulation disorder. First report of a case outside Friuli. Acta haemat., 49, 114-122.

13) Girolami, A., Molaro, G. \& DeMarco, L. (1974) Factor X survival and therapeutic factor $\mathrm{X}$ levels in the abnormal factor X (Factor X Friuli) coagulation disorder. Acta haemat., 52, 223-231.

14) Girolami, A., Coser, P., Brunetti, A.\& Prinoth, O. (1975) Classical factor X deficiency. Report of a further case. Acta haemat., 53, 118-127.

15) Gonzalo, G.B., Lucia, R.O., Alejandro, V.G. \& Pavlo, L.V. (1973) Deficiencia familiar de factor X. Sangre, 18, 178-185.

16) Haber, S. (1964) Norethynodrel in the treatment of factor $\mathrm{X}$ deficiency. Arch. intern. Med., 114, 88-94.

17) Hoerder, M.H. (1959) Differential diagnose des Kongenitalen Stuart-Faktor-Mangels. Klin. Wschr., 37, 443-445.

18) Holzknecht, F. (1965) Kongenitaler Stuart-Prower-Faktor (Faktor X) Mangel. Klin. Wschr., 43, 82-84.

19) Hougie, C., Barrow, E.M. \& Graham, J.B. (1957) Stuart clotting defect. I. Segregation of an hereditary haemorrhagic state from the heterogeneous group heretofore called stable factor (SPCA) deficiency. J. clin. Invest., 36, 485-496.

20) Johnson, J.F. (1957) Changes in plasma prothrombin, A-c globulin and antithrombin concentration following intravenous administration of estrogens. Proc. Soc. exp. Biol. Med., 94, 92-94.

21) Kekwick, R.A. \& Wolf, P. (1957) A concentrate of human antihaemophilic factor. Its use in six cases of haemophilia. Lancet, 1, 647-650.

22) Krieger, H.N., Hilgartner, M.W. \& Frank, R.S. (1977) Surgery in patients with congenital disorders of blood coagulation. Ann. Surg., 185, 290-297.

23) Kurz, R., Holzknecht, G.J. \& Spoettl, F. (1969) Isolierter familiaerer Faktor-XMangel (Nachweis des rezessiven Erbgangs). Schweiz. med. Wschr., 99, 885-888.

24) Lechler, E., Webster, W.P., Roberts, H.R. \& Penick, G.D. (1965) The inheritance of Stuart-Disease: Investigation of a family with factor $\mathrm{X}$ deficiency. Amer. J. med. Sci., 249, 291-294.

25) Lorraince, M., Goryea, M.S. \& William, K. (1958) Congenital coagulation deficiency of Stuart factor activity. J. Lab. clin. Med., 51, 398-409.

26) Macfarane, R.G., Biggs, R. \& Bidwell, E. (1954) Bovine antihaemophilic globulin in the treatment of haemophilia. Lancet, 1, 1316-1319.

27) McGovern, J.J., Bunker, J.P., Goldstein, R. \& Estes, J.W. (1961) Effects of conjugated estrogens on the coagulation mechanism. J. Amer. med. Ass., 175, 10111012.

28) Mori, K., Hiratsuka, I., Nakano, N. \& Naito, T. (1972) A case of congenital factor X defiicnecy. Jap. J. Clin. Hemat., 18, 635-636. (Japanese)

29) Mori, K., Sakai, H., Hiwatashi, K., Hasegawa, T., Gordon, L.S. \& Yamagata, S. (1975a) 
Two cases of congenital factor X deficiency. Third Meeting of the Asian Pacific Division of the International Society of Haemeatology, Jakarta.

30) Mori, K., Hiratsuka, I., Sakai, H., Hiwatashi, K., Hasegawa, T. \& Yamagata, S. (1975b) Effects of administration of factor II, VII, IX and $\mathrm{X}$ concentrate on hemorrhagic diathesis associated with liver disease. Med. Consult. new Remed., 12, 599-605. (Japanese)

31) Mori, K., Sakai, H., Hiwatashi, K. \& Hasegawa, T. (1976) A case of congenital factor X deficiency. 42nd Tohoku Ketsuekigaku Dohkohkai, Sendai.

32) Oehme, J., Scheick, G. \& Schultze, H.E. (1958) Familiaerer Faktor X-Mangel. Klin. Wschr., 36, 521-524.

33) Okamoto, E., Nakayama, H., Tachikawa, T., Wakizaka, K. \& Yasunaga, K. (1967) Turn-over and family study in a patient with congenital factor $\mathrm{X}$ deficiency. Acta haemat. jap., 30, 721-722. (Japanese)

34) Parkin, J.D., Madaras, F., Sweet, B. \& Castaldi, P.A. (1974) A further inherited variant of coagulation factor X. Aust. Newzeal. J. Med., 4, 561-564.

35) Pigeaud, H., Favre-Gilly, J., Legrat, J. \& Thouverez, J.P. (1964) Deficit congenital en facteur Stuart. Accouchement sans incident apres injection de fraction C.S.B. Bull. Fed. Soc. Gyn. Obst., 16, 556-557.

36) Rabiner, F. \& Kretchmer, N. (1961) The Stuart-Prower factor: Utilization of clotting factors obtained by starch block electrophoresis for genetic evaluation. Brit. $J$. Haemat., 7, 99-111.

37) Rizza, C.R. (1972) The clinical features of clotting factor deficiencies. In: Human Blood Coagulation, Haemostasis and Thrombosis, edited by R. Biggs, Blackwell Publishing Co., London-Melborne, pp. 210-229.

38) Roberts, J.R., Lechler, E., Webster, W.P. \& Penick, G.D. (1965) Survival of transfused factor $\mathrm{X}$ in patients with Stuart disease. Thrombos. Diath. haemorrh., 13, 305-313.

39) Roos, J., Van Arkel, C., Verloop, M.C. \& Jordan, F.L.J. (1959) A "new" family with Stuart-Prower deficiency. Thrombos. Diathes. haemorrh., 3, 59-76.

40) Sakai, H., Takeda, H., Tanaka, H., Yahata, J., Mori, K., Suzuki, S. \& Seino, Y. (1979) Hemostatic control of factor $\mathrm{X}$ deficiency during and after surgery. Iryo, 33, 771-775. (Japanese)

41) Sarup, B.M., Perkash, A. \& Aikat, B.K. (1972) Congenital Stuart Prower factor (Factor X) deficiency. J. Indian med. Ass., 59, 110-114.

42) Schneegans, E., Mayer, G., Burgun, P., Levy-Silagy, J., Kalogjera, V., Wiesel, M-L., Peter, J. -D. \& Pautler, J. (1976) Le facteur Stuart. A propos d'un cas deficit congenital. Pediatrie, 31, 17-31.

43) Shirakawa, M., Hyodo, S., Asayama, R., Anayama, N., Tomita, K., Nakahara, M. \& Miyamoto, N. (1969) A case of congenital factor X deficiency. Med.J. Mutual Ass., 18, 205-209. (Japanese)

44) Szönyi, L., Palos, T. \& Rentka, M. (1969) Mangel am kongenitalen Faktor X (Faktor Stuart-Prower). Z. Kinderheilk., 106, 100-106.

45) Telfer, T.P., Denson, K.W.E. \& Wright, D.R. (1956) A "new" coagulation defect. Brit. J. Haemat., 2, 308-316.

46) Yasunaga, K. Okamoto, E. \& Wakizaka, K. (1964) A case of congenital factor X deficiency. Acta haemat. jap., 27, 377 (Japanese). 